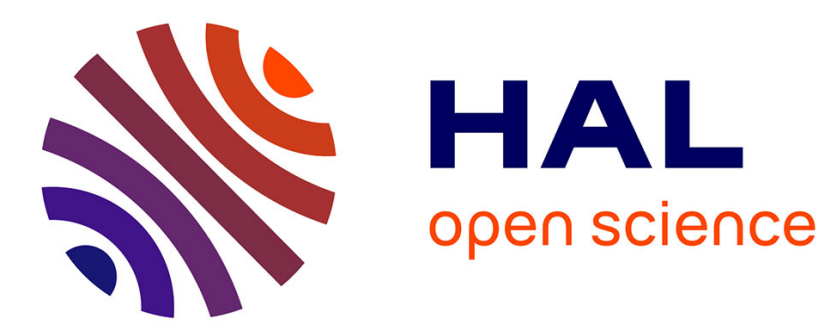

\title{
Poèmes taoïstes des cinq veilles
}

Vincent Goossaert

\section{To cite this version:}

Vincent Goossaert. Poèmes taoïstes des cinq veilles. Études Chinoises, 2000, 19 (1-2), pp.249-270. halshs-00188383

\section{HAL Id: halshs-00188383 \\ https://shs.hal.science/halshs-00188383}

Submitted on 16 Nov 2007

HAL is a multi-disciplinary open access archive for the deposit and dissemination of scientific research documents, whether they are published or not. The documents may come from teaching and research institutions in France or abroad, or from public or private research centers.
L'archive ouverte pluridisciplinaire HAL, est destinée au dépôt et à la diffusion de documents scientifiques de niveau recherche, publiés ou non, émanant des établissements d'enseignement et de recherche français ou étrangers, des laboratoires publics ou privés. 


\title{
Poèmes taoïstes des cinq veilles
}

\author{
Vincent Goossaert ${ }^{1}$
}

Les derniers cycles de cours de Jean-Pierre Diény à l'École pratique des Hautes Études étaient consacrés à la postérité de l'affirmation de Zhuangzi selon laquelle « le Saint ne rêve pas ${ }^{2}$. En hommage au professeur, je voudrais évoquer ici l'un des recoins les plus mal connus de cette abondante postérité. L'ordre taoïste Quanzhen, apparu vers 1170, a pratiqué de nombreuses formes d'ascétisme, et en particulier la privation de sommeil. Le but de cette épreuve était précisément de ne plus rêver, mettant ainsi par la force le précepte de Zhuangzi en application. Le combat nocturne contre la somnolence et la tentation des rêves était un exercice quotidien, dont plusieurs maîtres ont laissé des récits métaphoriques. Les œuvres poétiques Quanzhen contiennent une quinzaine de séries de « poèmes des cinq veilles (wugeng) ${ }^{3}$ », qui constituent des descriptions du travail sur soi-même aboutissant à l'immortalité, dans le cadre de ces nuits de méditation. Qu'un

1 Vincent Goossaert est Chargé de recherche au CNRS (FRE Religion et Société en Chine). L'auteur remercie Catherine Despeux pour ses remarques et corrections apportées aux traductions.

2 Guo Qingfan (éd.), Zhuangzi jishi, Pékin, Zhonghua shuju, 1961, p. 228.

$3 \mathrm{Au}$ sens de veille, le caractère geng connaît également une prononciation ancienne : jing. 
spécialiste de l'histoire sociale s'attaque à la poésie taoïste peut laisser craindre le pire ; je souhaite simplement ici faire connaitre une certaine tradition de poésie lyrique et m'interroger sur les rapports entre l'usage d'un genre poétique et la pratique ascétique. Au demeurant, $j$ 'admets dès l'abord que mes traductions sont très libres, et sans aucun doute perfectibles.

La tradition taoïste s'est toujours méfiée du sommeil. Selon le Huangting jing, «passer jour et nuit sans dormir permet de réaliser le Vrai » ${ }^{4}$. S'il est vrai que de nombreux taoïstes, en particulier Chen Tuan (?-989), sont de grands dormeurs ${ }^{5}$, ils n'en privilégient pas moins le contrôle permanent de soi. L'esprit (shen) doit rester jour et nuit maître de l'organisme, et surtout de son propre siège $(x i n)$ et de son activité $(y i)$; il doit en surveiller les actions et en limiter les échanges avec l'extérieur. De plus, les divinités qui contrôlent les hommes font des visites nocturnes, pendant lesquelles il convient d'être éveillé ${ }^{6}$. Dans l'ordre Quanzhen, cette méfiance devient une véritable obsession. On craint que les désirs, en particulier la libido, tenus fermement sous contrôle pendant la journée, ne se déchaînent la nuit, à la faveur des rêves. Les rêves érotiques et les émissions involontaires sont spécifiquement évoqués et redoutés ${ }^{7}$. Les textes, prescriptifs comme descriptifs, nomment démons ( $m o$ ) ces tentations, présentées comme autant d'épreuves. Le terme mo n'implique pas qu' on leur accorde une existence matérielle, et les auteurs sont conscients qu'il s'agit d'hallucinations nées d'un organisme malmené par l'ascèse. Dans le cas de visions éprouvées par le pratiquant en proie à la torpeur, il s'agit des shuimo, « démons du

4 Ce vers ne figure pas dans la version originale du Huangting jing, mais dans une version tardive du Ive siècle, au sixième vers du troisième paragraphe de la version « intérieure » (Huangting neijing yujing).

5 Sa méthode d'ascèse par le sommeil, shuigong, est souvent évoquée, mais nulle part détaillée.

6 Cf. Yunji qiqian 81.10b, Daozang 1032. Les ouvrages du Daozang sont donnés dans la numérotation établie par Kristofer Schipper, Concordance du Tao-tsang, Paris, École française d'Extrême-Orient, 1975.

7 Il existe plusieurs anecdotes à ce sujet dans les entretiens des maîtres Quanzhen ; voir par exemple Qinghe zhenren beiyou yulu 3.11b-12a (1237), Daozang 1310. 
sommeil ", qu'il faut vaincre en les sublimant (lian shuimo). Ce thème est très fréquent dans l'ensemble de la littérature taoïste de l'époque Jin-Yuan ${ }^{8}$.

Par ailleurs, il est depuis longtemps recommandé de faire usage des heures de la nuit pour méditer ; minuit, ou plutôt l'heure $z i$, est le moment du yang naissant et se révèle donc particulièrement favorable. Les heures, par leur décompte au moyen des douze branches, entrent dans le système de correspondances sur lequel joue le Neidan, l'« alchimie intérieure », la tradition spéculative sur laquelle est fondé l'ensemble des pratiques de méditation taoïstes à partir des Song, et que le Quanzhen a largement contribué à diffuser ${ }^{9}$. Le Neidan fournit le fondement théorique et une partie du vocabulaire de nos poèmes, et l'on y retrouve fréquemment le thème du yang naissant au milieu de la nuit. Les « poèmes des cinq veilles », cependant, utilisent assez peu le jeu symbolique mettant en rapport cycles du temps, trigrammes et procédures alchimiques, dans la mesure où ils sont rythmés par les veilles, indépendantes des heures. La durée des cinq veilles, en effet, n'est pas fixe : elle dépend de la longueur de la nuit ${ }^{10}$. Le choix des auteurs Quanzhen de compter en veilles plutôt qu'en heures s'explique certainement par leur association aux rondes militaires et à la garde, également présente dans le terme français de «veille». De même que les soldats, les taoïstes luttent contre le sommeil et gardent leur citadelle

8 Cf. Vincent Goossaert, La création du taö̈sme moderne : l'ordre Quanzhen, thèse de doctorat, École pratique des Hautes Études, Section des Sciences Religieuses, mai 1997, p. 148-149 et 197-200 (à partir de sources épigraphiques) ; Kubo Noritada, " Kindai no shin Dôkyô to Bukkyô - sankyô chôwa shisô kara mita », Tôhôgaku, 25, 1963, p. 37-38 (article réédité dans Mongoru chô no Dôkyô to Bukkyố - nikyô no ronsô wo chûshin ni, Tokyo, Hirakawa shuppansha, 1992, p. 9-30). Pour une discussion spéculative, voir « Bing shuimo wen » de Bai Yuchan, Xiuzhen shishu 42.5a-7b, Daozang 263. Par ailleurs, les expressions shuimo et lian shuimo se retrouvent dans des centaines de poèmes, anecdotes, inscriptions, etc., issus du Quanzhen.

9 Ce n'est pas le lieu ici d'exposer les fondements du Neidan. Les publications sur le sujet se multiplient depuis une dizaine d'années. Pour une approche d'ensemble, on consultera Isabelle Robinet, Introduction à l'alchimie intérieure taoiste. De l'unité et de la multiplicité, Paris, Le Cerf, 1995.

10 Les dictionnaires donnent souvent, à tort, une durée fixe de deux heures à la veille. 
intérieure de tout intrus. Par ailleurs, les cinq veilles sont marquées dans les monastères (taoïstes comme bouddhiques) par des coups de tambour. Les règles Chan prévoient même cinq intervalles (dian) divisant chaque veille; chacun de ces vingt-cinq intervalles est marqué par un nombre spécifique de coups sur une planche métallique (yunban). Les veilles constituent donc pour les communautés religieuses une réalité bien sensible.

Une autre motivation de la méditation nocturne se trouve dans la pratique communautaire : les novices sont appelés à travailler au bien commun durant la journée et à avancer leur propre recherche spirituelle durant les heures restantes. Les règles communautaires du Quanzhen prévoient des méditations alternées avec des lectures à haute voix, tout au long de la nuit, afin de chasser l'hébétude ${ }^{11}$. La réalité d'une telle pratique est évoquée dans de nombreuses biographies relatives aux maitres Quanzhen ; on trouve également dans les entretiens de Yin Zhiping (1169-1251), qui devint le puissant supérieur de l'ordre en 1227 , un discours sur la manière de la mettre en œuvre :

Commencez par diminuer le temps de sommeil durant la nuit, jour après jour, mois après mois, sans chercher de résultat rapide. Spontanément, les souffles de torpeur cesseront de surgir et vous obtiendrez graduellement la force de vous contrôler. Jadis, quand je demeurais au Shandong, je pratiquais de cette façon. Quand je me sentais fatigué, j'allais aussitôt m'activer à une tâche. Jour après jour, j'en vins à tenir ainsi jusqu'à vingt-quatre ou vingt-cinq jours. Cela devenait naturel et mon esprit s'en trouvait limpide. Vous tous, vous devez pratiquer cela ${ }^{12}$

Toutes les pratiques de méditation nocturne ne correspondent pas nécessairement à une privation extrême de sommeil, mais, dans le cas du Quanzhen, elles s'inscrivent souvent dans le cadre d'une discipline collective très exigeante, de même d'ailleurs que la méditation Chan dans les grands monastères bouddhiques. Pour les adeptes solitaires, sans doute les plus nombreux au sein du taoïsme, il n'existe pas d'obligation de veille : ils n'en pratiquent pas moins la méditation nocturne et ont pour idéal la réduction « naturelle» du sommeil.

11 Cf. Quanzhen qinggui 6a (début du xIV ${ }^{\mathrm{e}}$ siècle), Daozang 1235.

12 Qinghe zhenren beiyou yulu 1.1b-2a. 
L'expérience de nuits passées à méditer laisse une trace sous la forme de séries de «poèmes des cinq veilles », dont le présent article propose seize exemples trouvés dans la littérature taoiste des Jin et des Yuan, sans compter quelques séries plus tardives, qui ne sont pas prises en compte ici ${ }^{13}$. On trouvera en annexe une liste numérotée du corpus étudié auquel je renverrai désormais. Il ne saurait s'agir d'épuiser le thème de la nuit ascétique ; je me contenterai d'évoquer des poèmes répondant à des caractéristiques formelles bien précises, à savoir des séries de cinq pièces 14 commençant par « la première veille », " la deuxième veille », etc., ainsi qu'une variante, qui remplace la veille par le coup de tambour $(g u)$, utilisée à trois reprises (séries 2,5 et 6 ). Une seule de ces séries est en poésie régulière (shi, série 13), les autres étant des ci sur différents airs (cipai).

Avant de proposer deux traductions représentatives, puis quelques réflexions d'ensemble sur ces poèmes, je voudrais les présenter sous un angle bibliographique. Le corpus rassemblé, appartenant sans aucun doute à un ensemble plus important en partie perdu, semble constituer un sousgenre de la poésie Quanzhen. S'il est difficile de raisonner sur une absence, surtout à l'intérieur d'une littérature encore peu explorée, il me paraît significatif de n'avoir trouvé aucun exemple de « poèmes des cinq veilles »

13 Six séries intitulées "Wugeng daoqing », dans Zhang Sanfeng xiansheng quanji, Xuanyao pian xia 48b-56a, édition Daozang jiyao ; une autre série du même titre, Xuanyao pian xia 65a-66a ; et une série intitulée "Jiugeng daoqing", Xuanyao pian xia 56a-58a. La première de ces huit séries est éditée, sans indication de source, dans Xie Boyang (éd.), Quan Ming sanqu, Jinan, Qilu shushe, 1994, p. 233-234. Le personnage de Zhang Sanfeng est d'une historicité contestée ; l'anthologie Zhang Sanfeng a été compilée sous les Qing par Li Xiyue. On trouve également une série dans Liu Yiming (1734-1821), Huixin waiji 1.28b-29a, dans Daoshu shi'er zhong, édition de la fin des Qing, rééditée à Pékin par Zhongguo zhongyiyao chubanshe, 1990, p. 685.

14 Dans plusieurs cas (séries 1 et 4), on trouve en réalité sept poèmes, les cinq veilles étant encadrées par une sorte d'introduction et de conclusion. Ci-après, j'appellerai « suite » ou « série » un tel ensemble et «poème » ou «pièce » l'une de ses composantes. 
chez les auteurs contemporains des autres écoles taoïstes, en particulier dans celle du Nanzong (Tradition du Sud), lignée mal connue historiquement mais très prestigieuse qui laissa une importante production poétique ${ }^{15}$.

Par ailleurs, il est également à noter que six de nos séries sont présentes dans une anthologie de la poésie taoïste des Song et des Yuan où le Quanzhen occupe la meilleure place. Il s'agit du Minghe yuyin (Échos des chants des grues). Cette collection était à l'origine composée de douze poèmes lyriques du maître Quanzhen Feng (zi Changquan, milieu du XIII siècle), rendus célèbres en Chine du Sud par une chanteuse, auxquels l'éminent poète et homme d'État Yu Ji (1272-1348) composa une suite. Elle fut augmentée en 1347 de quelque cinq cents pièces (essentiellement des $c i$ ) par un maître taoïste, également de Chine du Sud, Peng Zhizhong, qui y rassembla des compositions apparentées au daoqing. Ces « sentiments taoïstes» forment un style lyrique, qui chante le détachement du monde et les joies de l'immortalité, thèmes également très présents dans le théâtre (zaju) de l'époque. La présence de six séries (rassemblées deux par deux, 9 et 7, 10 et 8,11 et 12) dans une anthologie qui semble avoir connu une importante diffusion suggère la popularité des « poèmes des cinq veilles » et leur place non négligeable à l'intérieur de la poésie Quanzhen.

Une autre caractéristique du corpus est la place qu'y occupent les premiers patriarches du Quanzhen, qui ont composé les pièces les plus anciennes et les plus nombreuses. En effet, la moitié du corpus est due au fondateur du Quanzhen, Wang Zhe (daohao Chongyang, 1113-1170, séries 1 à 4), et à son disciple Ma Yu (daohao Danyang, 1123-1184, séries 5 à $8)^{16}$. La production poétique de Wang a été éditée dans les années 1170 1180 , après sa mort, dans l'entourage de Ma. Sous sa forme actuelle, elle ménage une place très importante aux échanges poétiques entre le maître et son disciple favori. On y trouve en effet de très nombreuses pièces qui se

15 Le Nanzong se donne pour fondateur le mystérieux Zhang Boduan et son Wuzhen pian, l'une des ouvres de référence du Neidan. Il s'épanouit au XIII siècle, puis s'intègre graduellement au Quanzhen.

16 Sur la vie et l'œuvre poétique de Wang Chongyang et Ma Danyang, voir surtout Hachiya Kunio, Kindai Dôkyô no kenkyûu - O Chôyồ to Ba Tanyô, Tokyo, Tôkyô daigaku Tôyô bunka kenkyûjo hôkoku, Kyûko shoin, 1992. 
répondent. Cependant les huit poèmes nocturnes qui nous intéressent semblent tous indépendants.

L'une des particularités des poèmes de Wang Chongyang est l'usage de deux airs spécifiquement prévus pour les «poèmes des cinq veilles », Wugeng chu shelang et Wugeng ling. Ces cipai assez rares ${ }^{17}$ semblent bien avoir été inventés pour l'occasion par Wang, à moins qu'il n'ait renommé ainsi un cipai déjà existant, une pratique courante chez les auteurs taoïstes. Ils pourraient également avoir été inspirés par plusieurs cipai intitulés Wugeng zhuan, de rythme différent, mais également utilisés pour des suites de cinq poèmes qui ont été retrouvées à Dunhuang (composées au I $\mathrm{X}^{\mathrm{e}}$ ou au $\mathrm{X}^{\mathrm{e}}$ siècle ?) ${ }^{18}$. Parmi ces dernières, on trouve des ballades amoureuses ${ }^{19}$, mais on remarque surtout une dizaine de séries bouddhiques, dont cinq portent sur la méditation et décrivent un éveil progressif ${ }^{20}$. S'ils sont plus métaphysiques et moins portés vers la transformation du corps que les œuvres Quanzhen, les poèmes nocturnes de Dunhuang n'en forment pas moins un précédent remarquable et direct au corpus étudié ici ${ }^{21}$. En bonne

17 On ne les trouve pas dans le (Yuzhi) Cipu (1715).

18 Cf. Pan Shen et al., Cilï cidian, Taiyuan, Shanxi renmin chubanshe, 1991, p. 1192-1200. Voir aussi une brève note dans Wong Shiu-hon (Huang Zhaohan), «Quanzhen jiaozu Wang Chongyang de ci », in Daojiao yanjiu lunwen ji, Hong Kong, The Chinese University Press, 1988, p. 199-200 (première édition dans Dongfang wenhua, 19.1, 1981, p. 29-43). Le même auteur évoque également ces poèmes dans son « Quanzhen qizi ci pingshu », Daojiao yu wenxue, Taipei, Taiwan xuesheng shuju, 1994, p. 43-85 (première édition dans Xianggang zhongwen daxue Zhongguo wenhua yanjiusuo xuebao, 19, 1988, p. 135-162), p. 62-63.

19 Des poèmes des cinq veilles sans connotation religieuse, galants ou nostalgiques, continueront à être composés par la suite ; on en trouve notamment dans la ballade libre (sanqu) d'époque Ming.

20 Cf. Ren Bantang (comp.), Dunhuang geci zongbian, Shanghai, Shanghai guji chubanshe, 1987, p. 1225-1580. Une partie est reproduite dans Zhang Zhang et Huang She (comp.), Quan Tang Wudai ci, Shanghai, Shanghai guji chubanshe, 1986, p. 931-938.

21 On trouve une ressemblance formelle entre la série 16 et certaines des séries de Dunhuang (notamment Dunhuang geci zongbian, p. 1284), où chacun des cinq 
partie d'inspiration Chan, ces « poèmes des cinq veilles » font partie d'un ensemble plus large de séries à structure répétitive, comportant par exemple les « poèmes des douze heures », des « douze mois » ou des « cent années de la vie $»$. Remontant plus loin dans le temps, l'origine première du genre semble se trouver dans une série de cinq poèmes réguliers due à Fu Zhidao (fin du vi ${ }^{\mathrm{e}}$ siècle), intitulée «Congjun wugeng zhuan » (La succession des cinq veilles, pendant une campagne militaire), ce qui confirme le lien entre la garde militaire et la veille religieuse ${ }^{22}$. La tradition d'un cipai ancien, spécifique au genre des «cinq veilles », n'a pas pour autant joui d'une postérité notable au sein du Quanzhen, puisque aucun autre auteur de mon corpus n'a utilisé ces airs.

Le genre des " poèmes des cinq veilles » ne semble pas avoir intéressé les autres disciples de Wang Chongyang. On le retrouve en revanche chez les auteurs de la seconde génération, dont Yin Zhiping (série 13), évoqué plus haut, et chez deux auteurs mal identifiés cités par le Minghe yuyin (séries 9 et 10). Dans le même ouvrage sont incluses deux séries (11 et 12) attribuées à Lü Dongbin. L'historicité de cet immortel, saint patron du Quanzhen, restera à jamais débattue, mais beaucoup de poèmes furent composés en son nom à partir du $x_{1}^{\mathrm{e}}$ siècle, y compris par l'écriture inspirée. Les séries 14 et 15 sont dues à un auteur de la fín des Yuan, Wang Jie. Sa carrière est mal connue, mais ses œuvres sont un bon exemple de la fusion du Quanzhen et du Nanzong. La dernière série, enfin, semble poser un problème d'authenticité. Elle est attribuée au célèbre Zhang Jixian (10921126), le $30^{\mathrm{e}}$ Maître céleste, qui œuvra à la cour de l'empereur Song Huizong (r. 1100-1125). Zhang est fréquemment cité dans la littérature taoïste à partir de sa mort, mais son anthologie littéraire ne fut compilée qu'en 1395 par l'éminent bibliographe Zhang Yuchu (1361-1410), lui-même 43 ${ }^{\mathrm{e}}$ Maître céleste. Plusieurs poèmes de cette collection sont explicitement posthumes, ce qui permet de supposer qu'une partie, dont peut-être la série 16, est en

poèmes commence par une phrase de trois caractères qualifiant la veille dont il est question : yigeng chu, ergeng shen, etc.

22 Cf. Lu Qinli (comp.), XianQin Han Wei Jin Nanbei chao shi, Pékin, Zhonghua shuju, 1983, p. 2602-2603. 
réalité d'une plume plus tardive ${ }^{23}$. Cette suite est la seule du corpus à n'avoir aucun lien apparent avec l'ordre Quanzhen.

La première traduction que je propose (série 5, de Ma Danyang) est rythmée non par les cinq veilles, mais par les cinq coups de tambour qui les annoncent. L'unité de la série est fortement renforcée par une phrase cinq fois identique au centre de chaque poème. Chacune des premières phrases est également identique ; Ma Danyang affirme y dormir (mian), mais, au vu du reste des poèmes, il faut sans doute comprendre qu'il se trouve dans un état de calme profond où la conscience reste active (un tel usage du terme mian est attesté dans la littérature Quanzhen ${ }^{24}$ ) :

Au premier coup du tambour, je « dors » seul et me retiens.

D'un nuage, je laboure mon précieux terreau

Et je plante dru les pousses du champignon pourpre.

Dès lors, mon paradis intérieur ne souffre plus de fuite,

Plus de perte, plus d'écoulement.

Le tigre-kan et le dragon-li s'embrassent ${ }^{25}$.

Au deuxième coup du tambour, je « dors » seul et me retiens.

Les nuages se déchirent sur un ciel constellé,

Où soleil et lune échangent leur éclat.

Dès lors, mon précieux vase ne souffre plus de fuite,

Plus de perte, plus d'écoulement.

Dragon et serpent sortant de terre engagent le combat.

23 Le Quan Song ci se fonde non sur le Daozang, mais sur une anthologie Ming dont j'ignore les sources.

24 Par exemple «méditer en oubli au point d'être comme quelqu'un qui dort », zuowang mian, dans l'inscription Da Yuanguo Dadu lu Yizhou Laishui xian Tongting xiang Tanshan li Tongxuan dashi chuangjian Tianzhen guan ji, de Liu (caractère lacunaire)gui, estampage de la Collection Yifeng tang (bibliothèque de l'Université de Pékin), transcrit dans Chen Yuan (1880-1971, comp.), Chen Zhichao et Zeng Qingying (éds), Daojia jinshi lüe, Pékin, Wenwu chubanshe, 1988, p. $622-623$.

25 Les deux trigrammes $k a n$ et $l i$, ainsi que dragon et tigre, plomb et mercure, jouvencelle et enfançon, font partie des couples utilisés de multiples manières par le Neidan. Leur union symbolique permet d'avancer dans la création d'un embryon d'immortalité. En aucun cas ils ne renvoient à une réalité physique unique. 
Au troisième coup du tambour, je « dors » seul et me retiens.

Je frappe à coups redoublés à la porte du mystère

Et réveille à grands cris celui qui s'y trouve enfermé.

Dès lors, le verrou de jade ne souffre plus de fuite,

Plus de perte, plus d'écoulement.

La demoiselle et l'enfançon se prennent par la main.

Au quatrième coup du tambour, je « dors » seul et me retiens.

Par d'infinies précautions je repousse les voleurs,

Et ma sagesse (prajna) vigilante met les démons en déroute.

Dès lors, ma moelle d'or ${ }^{26}$ ne souffre plus de fuite,

Plus de perte, plus d'écoulement.

Une goutte de lumiere toute-puissante se forme.

Au cinquième coup du tambour, je " dors » seul et me retiens.

Dans une parfaite sérénité la présence surgit au cœur de l'absence ;

Je participe au monde en restant dans l'immuable.

Dès lors, mon cinabre divin ne souffre plus de fuite,

Plus de perte, plus d'écoulement.

Apparaît dans sa splendeur mon embryon d'immortel.

La seconde traduction est celle de la série 9, tirée du Minghe yuyin, attribuée à un certain Yang zhenren que les éditeurs du Quan Jin Yuan ci identifient à Yang Mingzhen (1150-1228), un disciple de Ma Danyang, connu par plusieurs sources épigraphiques. Même s'il est sans doute le plus célèbre des taoïstes d'époque Jin-Yuan ayant porté ce patronyme, et si sa relation à Ma Danyang plaide pour cette identification, celle-ci devra rester une simple hypothèse qui ne gêne en rien la lecture des poèmes :

À la première veille, je saisis le cheval de l'imagination ${ }^{27}$,

Le gibbon, quant à lui, n'est plus libre de faire le fou ${ }^{28}$.

26 Jinjing, l' « essence séminale » associée à la phase « métal ».

27 Le cheval de l'imagination (yima) et le gibbon de l'esprit (xinyuan) sont deux métaphores omniprésentes dans la littérature taoiste des Jin et des Yuan. De nature folâtre, ils nécessitent un sévère dressage, préalable à l' ascèse alchimique.

28 Dian*, c'est-à-dire prendre les choses pour leur contraire et l'illusoire pour réel. 


\section{Poèmes taoïstes des cinq veilles}

Le grand éveil survient : mon esprit s'ouvre à la pure fraîcheur, Spontanément, je lâche prise et me détache de tout.

Mon visage originel immuablement distingué,

Dans le Pur calme je m'enfonce dans une subtile obscurité.

Je ne me sers plus de ma bouche, mais je nourris le souffle et

l'âme à satiété.

En suivant le rythme du temps, je dissèque la création.

À la deuxième veille, les deux époux s'unissent,

L'enfançon et la demoiselle se rencontrent.

Le haut et le bas sont inversés ${ }^{29}$; l'espace vide renversé tourne sur luimême,

Je saisis kan et li qui s'assemblent l'un à l'autre.

Le bœuf-terre et le cheval-bois agitent monts et mers

Que le mouton hisse le long de la colonne ${ }^{30}$.

Les flammes débordantes se précipitent dans le niwan ${ }^{31}$.

Je donne mes instructions aux esprits qui tremblent.

À la troisième veille, racines et branches s'affermissent, "Linglong », on fête aujourd' hui le double yang.

Je veux m'en retourner, mais la voiture se renverse dans la boue ;

C'est le moment d'agiter le terreau.

J'ai tranché entre bois et métal : corbeau et lièvre en jaillissent,

Le remède est prêt, plomb et mercure sont assemblés.

La neige blanche qui couvre le sol irrigue le bourgeon jaune ${ }^{32}$, Je contemple la fleur de jade, les pousses sortent du Lotus d'or.

29 Littéralement, les hexagrammes tai et fu.

30 Le char tiré par le mouton est l'un des trois véhicules à l'intérieur du corps, avec le char du bouf et celui du cerf.

31 Le niwan, terme ancien dont l'origine est mal connue, désigne généralement le cerveau. On le retrouve à la première veille de la série 8 .

32 Neige blanche (baixue) et bourgeon jaune (huangya) sont deux termes associés, notamment dans la tradition du Wuzhen pian. Ils renvoient le premier au mercure et le second au plomb, mais déjà mutuellement fécondés, si bien que leur rencontre (le premier descend et le second monte) est le moment où se forme l'embryon d'immortalité. Le bourgeon jaune, ici évoqué à la troisième veille, est plus généralement mentionné à la deuxième (séries $3,4,6$ ). 


\section{Vincent Goossaert}

À la quatrième veille, le tambour de l'office résonne,

Le coq d'or chante à l'unisson de la claquette.

Je dirige [le mouvement intérieur qui] tourne, monte et descend, franchit

Et aussitôt traverse la chambre des dieux ${ }^{33}$.

la double passe,

Le dragon et le tigre circulent à leur aise,

Je dose le feu et l'eau, je règle leur révolution.

Je raffine le noir et le rouge dans le chaudron, qui déverse dans le niwan. Balayant la neige blanche, je trouve un grain de cinabre.

À la cinquième veille, le ciel va s'éclairer, Mes travaux sont au complet, mon cuure est achevée.

Je veux quitter mon enveloppe, me mouvoir sans limite, Et revêtir ma figure authentique.

La famille des immortels, éternellement en liesse, Confere longue vie et abolit la vieillesse.

La convocation de l'empereur de Jade descend sous forme d'un édit pourpre, Chevauchant les nuages, je retourne aux îles Penglai.

Tous ces poèmes ont un style spécifique ; sans doute trouvera-t-on Ma Danyang plus lyrique et le zhenren Yang plus étroitement attaché à la rhétorique du Neidan, ce qui le rend plus difficile à lire en traduction. Une traduction complète du corpus serait fastidieuse, mais il est permis de proposer quelques réflexions nées d'une lecture comparée des seize séries. En premier lieu, on observe qu'elles ne présentent aucun parallélisme fort. On trouve certes des images qui reviennent dans une majorité d'entre elles : le pratiquant assis en méditation à la première veille, qui commence par calmer son esprit et régulariser son souffle ${ }^{34}$, la lune pleine au sommet de la voûte céleste à la troisième, le coq qui chante (à la quatrième ou la cinquième veille), l'aurore qui paraît. Les mêmes métaphores se retrouvent

33 Le mingtang est un des nombreux lieux abstraits utilisés par le Neidan qui ne correspondent pas à une réalité physiologique précise. La traversée du mingtang est également évoquée à la cinquième veille de la série 1 .

34 La série 13 précise que l'exercice commence dès la fin du repas vespéral, wancan. La série 16 précise que l'auteur est assis les jambes croisées (jiafu), sur un coussin de méditation (putuan), face à une lampe. 
fréquemment, surtout sur la lumière : pâleur lunaire (menglong) ou éclat de l'aurore (xiaguang). De manière générale, la lumière extérieure est un fil conducteur dans la plupart des suites.

En ce qui concerne les métaphores du corps, en revanche, si certaines expressions se retrouvent souvent d'une série à l'autre, elles le sont à des moments différents. Par exemple, l'apparition de la perle brillante (mingzhu, parfois perle précieuse, baozhu ou zhenzhu), esquisse d'un corps immortel, peut se retrouver à chacune des cinq veilles ${ }^{35}$. Ce résultat ne doit pas trop surprendre. Si les fondements théoriques du Neidan sont strictement définis, son usage laisse une très grande part à la créativité, y compris à la créativité littéraire. Une lecture comparée montre bien la difficulté de systématiser les processus du Neidan entre différents auteurs, voire même entre différents textes d'un même auteur.

Une systématisation est d'autant plus délicate que les séries de « poèmes des cinq veilles » ne sont pas à proprement parler des récits objectifs des nuits passées en méditation. Elles sont tout autant des évocations, rapides, allusives, de l'ensemble de l'œuvre alchimique depuis les premières étapes de purification jusqu'à l'apothéose finale. Au demeurant, les auteurs se réferent volontiers à d'autres parcours qui, comme la nuit divisée en cinq veilles, sont des métaphores de l'ensemble d'un itinéraire spirituel. C'est ainsi que plusieurs séries empruntent des images au dressage du buffle ${ }^{36}$.

On ne saurait nier, en revanche, que les différentes séries ont en commun d'être des traces d'une même expérience que l'on peut qualifier d'ascétique. On ne trouve certes pas dans les deux séries traduites plus haut, ni dans les autres, la peur panique du sommeil et des rêves évoquée au début. Plusieurs d'entre elles parlent même d'un demi-sommeil, comme la série 5 due à Ma Danyang, voire, dans deux cas, d'un bref sommeil léger à la quatrième veille ${ }^{37}$. Par ailleurs, la nécessité de repousser l'envie de dormir est

35 Première veille (série 10); deuxième (série 7) ; troisième (série 11); quatrième (séries 2 et 9); cinquième (séries 1 et 6 ).

36 Par exemple, deuxième veille de la série 12 . Sur la création poétique autour du thème du dressage du buffle, voir Catherine Despeux, Le chemin de l'éveil, Paris, L'Asiathèque, 1981.

37 Séries 6 et 13. 
nettement affirmée ${ }^{38}$. Le méditant continue toujours à surveiller son monde intérieur ; ainsi la série 16 utilise l'image d'une ville murée qui se ferme à la première veille pour ne s'ouvrir qu'à la cinquième. Signe qui ne trompe pas, on y parle fréquemment des démons, ou parfois des voleurs (une métaphore plus bouddhisante), qu'il faut chasser. C'est à la deuxième veille que leur expulsion est la plus fréquente (séries $2,9,11,13,15)^{39}$, ce qui suggère que c'est le moment où l'hébétude est la plus forte. Les poèmes de Ma Danyang, quant à eux, rappellent explicitement le rapport qui lie le désir sexuel et le sommeil ; la série 5, traduite plus haut, est rythmée par la phrase «sans écoulement» (wulou), tandis que la série 6, qui la suit immédiatement, qui est construite sur le même modèle, répète «sans rêve » (wumeng).

Les «poèmes des cinq veilles ", en tant que produits de la pratique formalisée sinon institutionnalisée qui consiste à méditer la nuit, posent naturellement le problème de la relation qu'entretient le genre poétique avec l'expérience mystique et l'identité du groupe d'adeptes. Même si les mâtres Quanzhen, parfois fins lettrés mais rarement esthètes oisifs, héritaient d'une tradition de poèmes nocturnes plus anciens ${ }^{40}$, ils ne sauraient avoir écrit ces séries pour le seul plaisir. La grande majorité des cuvres poétiques de l'ordre Quanzhen, dont il subsiste quelque dix mille pièces, sont de circonstance, composées en réponse à une question d'un disciple ou en guise d'encouragement. Il est donc probable qu'une partie des suites du présent corpus était donnée aux novices qui apprenaient à passer leurs nuits en méditation, ou même était destinée à être lue en public lors des nuits de méditation collective.

38 « Il faut avant tout réduire son sommeil ; lors du sommeil l'esprit se disperse et se trouve [sans protection] à l'air libre », première veille, série 7 .

39 Elle peut également avoir lieu à la première veille (séries 6,10 et 12) ou à la quatrième (séries 5 et 13). On retrouve l'expulsion des démons dans deux des séries de Dunhuang : Dunhuang geci zongbian, p. 1424 et 1429, dans les deux cas à la troisième veille.

40 Outre les poèmes de Fu Zhidao évoqués plus haut, on trouve dans la poésie non religieuse des séries de cinq poèmes nocturnes qui ne commencent pas par le compte des veilles et ne répondent donc pas à ma définition stricte. 
De fait, certaines ont un ton didactique, comme les séries 14 et 15 dues à Wang Jie. La série 4 est introduite par un poème qui présente l'ensemble comme des instructions (xiuxing jue). La plupart, cependant, ressemblent davantage au récit d'une expérience personnelle, peut-être même mise par écrit dès la fin de la nuit. Les suites les plus imagées, notamment celles de Wang Chongyang, où les termes associés à la vision sont prédominants ${ }^{41}$, laissent même entendre qu' afin de lutter contre les mo et les hallucinations démoniaques les ascètes créent leurs propres visions, paradisiaques mais tout aussi surprenantes. Celles-ci, et ce n'est pas le moindre paradoxe des poèmes des cinq veilles, sont parfois présentées comme des rêves (éveillés).

Ainsi, dans la série 12 :

Réveillé, je sens la pâleur lunaire apportée par le vent frais ;

Je réalise que j'entre dans la grotte de la source aux pêchers ${ }^{42}$, dans le jardin de paradis.

Je cherche à mon aise les trois seigneurs Mao ${ }^{43}$, je m'élève sans limite. Le vent passe par la fenêtre,

Je me rends compte en sursaut que je rêve mon voyage parmi les immortels ${ }^{44}$.

Le bon rêve, fabriqué, guidé, chasse le mauvais, qui est un pur produit de l'instinct ; le premier est contrôlé, tandis que le second est imprévisible. Dans quelle mesure ces visions oniriques contrôlées sont-elles fondées sur des données objectives, notamment des textes poétiques, narratifs ou didactiques?

Seule une comparaison systématique avec l'immense ensemble de la littérature Neidan permettrait d'établir dans quelle mesure chacune des visions relatées par les poèmes est partagée et transmise à l'intérieur de la

41 La série 3 accorde quant à elle une place importante aux sensations auditives, notamment au travers des coups de tambour qui annoncent chaque veille.

42 Cela renvoie au célèbre rêve de Tao Yuanming.

43 Les trois frères Mao sont les divinités du Maoshan (près de Nankin) et les saints patrons de la tradition Shangqing, qui s'y est développée à partir $\mathrm{du}_{\mathrm{I}} \mathrm{V}^{\mathrm{e}}$ siècle.

44 Meng you xian. Ce thème, qui a inspiré bien des compositions poétiques, notamment à l'époque Tang, se retrouve à la cinquième veille de la série 7 . 


\section{Vincent Goossaert}

tradition ou au contraire originale à l'auteur. Il est également permis de se demander si certaines de ces visions recrées n'avaient pas un support visuel ${ }^{45}$. La part de l'apprentissage (par l'enseignement oral du maître et par la lecture) et celle de la création spontanée, en des conditions difficiles ${ }^{46}$, sont malaisées à départager. L'une des visions les plus originales, tirée de la série 2 , peut se traduire comme suit :

Chevauchant un tigre, un jeune garçon;

À sa ceinture un couteau blanc, dans sa main une dague noire.

Il chasse cent démons, extermine mille démons, anéantit dix mille démons. Je me trouve au sein d'une forêt inextricable,

Qui crache de tous côtés des aiguilles d'argent.

On est tenté de juger un tel passage comme le fruit d'une méditation originale, spontanée. Cependant, la série à laquelle il appartient est très structurée, puisque chaque veille est marquée par l'une des cinq phases (wuxing) qui en détermine le ton ; la deuxième veille, dont la traduction cidessus est tirée, est clairement sous le signe de la phase métal, jin (tigre, couteau, dague, aiguilles). Dans la série 4, également très riche en images, chaque veille est associée à une direction. Dans le cours de la méditation, le flot des visions est guidé, car rien ne doit être laissé entièrement à la liberté de l'adepte dans un ordre comme le Quanzhen, où l'encadrement est toujours étroit.

Le lecteur moderne éprouve donc l'impression que les maîtres donnent aux disciples, aussi bien dans les instructions orales que dans les récits de

45 Si la littérature Neidan fournit surtout des schémas abstraits (en très grand nombre), on y trouve également des représentations figuratives. À titre d'exemple, le Yuqing jinsi qinghua biwen jinbao neilian danjue 2.13a, Daozang 240 , présente une image de la tortue au fond de l'océan qui aspire la lune, une métaphore que l'on retrouve dans les séries 10 (troisième veille) et 11 (quatrième veille).

46 On trouve déjà dans le taoïsme ancien des visualisations à pratiquer dans un état d'hallucination dû au manque de sommeil ; voir par exemple Stephen Eskildsen, Asceticism in Early Taoist Religion, New York, State University of New York Press, 1998, p. 49. 
leurs propres expériences consignées poétiquement, des thèmes, des schémas de progression spirituelle, qui peuvent scander leur nuit. Sans doute les maîtres donnent-ils également des motifs précis de visualisation que les disciples développent. Ceux-ci, à leur tour, consignent leur expérience par écrit et contribuent à l'élaboration d'un ensemble de textes - le genre des « poèmes des cinq veilles » - servant d'exemple et de référence. L'intérêt et la participation d'un large public à cet exercice (car la pratique de la méditation n'est pas le privilège d'une élite monastique) expliquent sans doute le succès et la diffusion des poèmes en dehors des cercles strictement religieux, comme le montrait jadis la diffusion des ballades bouddhiques de Dunhuang et comme le montre encore sous les Yuan l'histoire du Minghe yuyin, adopté par des lettrés et chanté en public.

Le résultat de cette création continue ne constitue pas un système, au contraire des « grands textes » de l'alchimie qui ont été plus souvent étudiés. Chacune des phrases des « poèmes des cinq veilles » pourrait être glosée par les classiques alchimiques, mais, prises ensemble, elles ne forment pas pour autant un discours cohérent, fini. Comme la plupart des genres poétiques, elles invitent à l'imitation plutôt qu'à l'interprétation. De même que chaque adepte éprouve ses propres tentations et difficultés particulières, il puise dans la littérature commune son propre moyen de les surmonter et relate à son tour son expérience sur le modèle prédéfini. Ainsi naît un genre qui donne aux adeptes de l'ordre religieux une manière d'intégrer à une expérience collective ce qui par définition est unique et incommunicable. 


\section{Liste des séries de poèmes}

La source première est donnée en-tête ; les éditions modernes les plus utiles sont citées entre parenthèses.

1 Wang Zhe (daohao Chongyang, 1113-1170), sept ci sur l'air Wugeng chu shelang, Chongyang Quanzhen ji 7.7b-8a (Quan Jin Yuan ci, p. 202203 ; Hachiya Kunio, 1992, p. 424).

2 Wang Zhe, cinq $c i$ sur l'air Xingxiang zi, Chongyang Quanzhen ji 8.5a6a (Quan Jin Yuan ci, p. 210-211 ; Hachiya Kunio, p. 429-430).

3 Wang Zhe, cinq ci sur l'air Wugeng ling, Chongyang Quanzhen ji 8.10a11a (Quan Jin Yuan ci, p. 213-214 ; Hachiya Kunio, p. 431-432).

4 Wang Zhe, sept $c i$ sur 1'air Chuanbo zhao, Chongyang Quanzhen ji 13.17b-18a (Quan Jin Yuan ci, p. 251 ; Hachiya Kunio, p. 472).

5 Ma Yu (daohao Danyang, 1123-1184), cinq ci sur l'air Wumeng ling, intitulés « Cinq veilles, envoi à l'ermite Zhao » («Wugeng ji Zhao jushi »), Jianwu ji xia 9b-10a (Quan Jin Yuan ci, p. 331 ; Hachiya Kunio, p. 546).

$6 \mathrm{Ma} \mathrm{Yu}$, cinq $c i$ sur l'air Wumeng ling, intitulés "Cinq veilles" («Wugeng»), Jianwu ji xia 10a-b (Quan Jin Yuan ci, p. 331-332; Hachiya Kunio, p. 546).

7 Ma Yu, cinq ci sur l'air Liangzhi yan'er, Minghe yuyin 6.7b-8b (Quan Jin Yuan ci, p. 397 ; Hachiya Kunio, p. 671).

$8 \mathrm{Ma} \mathrm{Yu}$, cinq ci sur l'air Gua jinsuo, Minghe yuyin 6.12a-13a (Quan Jin Yuan ci, p. 397-398 ; Hachiya Kunio, p. 671).

9 Yang zhenren (Yang Mingzhen ?), cinq ci sur l'air Gun jinwan, Minghe yuyin 6.6a-7b (Quan Jin Yuan ci, p. 1250-1251).

10 Fan zhenren (Fan Yuanxi ?), numéros 2 à 6 d'une série de $10 \mathrm{ci}$ sur l'air Guajinsuo, Minghe yuyin 6.10a-11a (Quan Yuan sanqu, p. 1950-1951). 
11 (Attribués à) Lü Dongbin, cinq $c i$ sur l'air Wutong shu, Minghe yuyin 7.11a-12a (Quan Jin Yuan ci, p. 1309-1310; Quan Tang Wudai ci, p. 1033-1034).

12 (Attribués à) Lü Dongbin, cinq $c i$ sur l'air Bubu gao, Minghe yuyin 7.12a-13a (Quan Jin Yuan ci, p. 1300 ; Quan Tang Wudai ci, p. 10341035).

13 Yin Zhiping (1169-1251), cinq poèmes réguliers intitulés « Chant de l'ascèse des cinq veilles » (« Xiuxing wugeng song »), Baoguang ji 1.9a-b.

14 Wang Jie (XIv e siècle), cinq $c i$ sur l'air Jinzi jing, Huanzhen ji 3.41b42a (Quan Yuan sanqu, p. 1948 ; Quan Yuan qu, p. 8690-8692).

15 Wang Jie, cinq $c i$ sur l'air Gua jinsuo, Cui gong Ruyao jing zhujie 15b16a (Quan Jin Yuan ci, p. 1266 ; Quan Yuan sanqu, p. 1948-1949; Quan Yuan qu, p. 8692-8693).

16 Zhang Jixian (1092-1126), cinq $c i$ sur l'air Du qingxiao, Sanshidai tianshi Xujing zhenjun yulu 6.14a-15a (Quan Song ci, p. 763-764).

\section{Sources des poèmes}

Baoguang ji, Daozang 1146.

Chongyang Quanzhen ji, Daozang 1153.

Cui gong Ruyao jing zhujie, Daozang 135.

Huanzhen ji, Daozang 1074.

Jianwu ji, Daozang 1142.

Minghe yuyin, Daozang 1100.

Quan Jin Yuan ci, Tang Guizhang (comp.), Pékin, Zhonghua shuju, [1979], 1992.

Quan Song ci, Tang Guizhang (comp.), Pékin, Zhonghua shuju, [1940], 1977.

Quan Tang Wudai ci, Zhang Zhang et Huang She (comp.), Shanghai, Shanghai guji chubanshe, 1986.

Quan Yuan qu, Xu Zheng et al. (comp.), Shijiazhuang, Hebei jiaoyu chubanshe, 1998.

Quan Yuan sanqu, Sui Shusen (comp.), Pékin, Zhonghua shuju, [1964], 1981. 


\section{Caractères chinois}

baixue 白雪

Bai Yuchan 白玉蟾

Baoguang $j i$ 菒光集

baozhu 賔珠

Bing shuimo wen 屏睡魔文

Bubu gao 步步高

Chen Tuan 陳摶

Chongyang quanzhen $j i$

重陽全真集

Chuanbo zhao 川撥棹

cipai 詞牌

Congjun wugeng zhuan 從軍五更轉

Cui gong Ruyao jing zhujie

崔公入藥經註解

Da Yuanguo Dadu lu Yizhou

Laishui xian Tongting xiang

Tanshan li

Tongxuan dashi

chuangjian Tianzhen

guan ji

大元國大都路易州淶水

縣通亭楖檀山里通玄大

師創建天鿓觀記

daoqing 道情

Daoshu shi 'er zhong

道書十二種

dian 點

dian*顛

Du qingxiao 度清霍

ergeng shen 二更深

Fan zhenren 范真人 (Fan

Yuanxi 范圓義 ?)
Feng 馮 (zi Changquan

長筌)

Fu Zhidao 伏知道

gu 鼓

Gua jinsuo 掛金索

Gun jinwan 輥金丸

Huanzhen ji 還眞集

Huangting jing 黃庭經

Huangting neijing yujing

黃庭内景王經

huangya 黃芽

Huixin waiji 會心外集

jiafu 跏蚗

Jianwu ji 漸悟集

jin 金

jinjing 金精

Jinzi jing 金字經

Jiugeng daoqing 九更道情

kan 坎

li 離

Li Xiyue 李西月

lian shuimo 鍊睡魔

Liangzhi yan'er 兩隻雁兒

Liu (caractère lacunaire) gui 劉口圭

Liu Yiming 劉一明

Lü Dongbin 呂洞賓

Ma Yu 馬鈺 (daohao

Danyang 丹陽)

Mao 茅

Maoshan 茅山

menglong 矇瞕

meng you xian 夢游仙 
mian 眠

Minghe yuyin 鳴鶴餘音 mingtang 明堂

mingzhu 明珠

mo 魔

Nanzong 南宗

Neidan 内丹

niwan 泥丸

Peng Zhizhong 彭致中

putuan 蒲團

Qinghe zhenren beiyou yulu

清和真人北游語錄

Quanzhen 全真

Quanzhen qinggui

全自清規

sanqu 散曲

Sanshidai tianshi Xujing zhenjun yulu

三十代天師虛靖貞君語錄

Shangqing 上清

shen 神

shuigong 睡功

shuimo 睡魔

wancan 晚餐

Wang Jie 王玠

Wang Zhe 王喜 (daohao

Chongyang 重陽)

wugeng 五更

Wugeng chu shelang

五更出舍郎

Wugeng daoqing

五更道情

Wugeng ji Zhao jushi

五更寄趙居士

Wugeng ling 五更令

Wugeng zhuan 五更轉

wulou 無漏

wumeng 無夢
Wumeng ling 無夢令

Wutong shu 梧桐樹

wuxing 五行

Wuzhen pian 悟䔔篇

xiaguang 霞光

xin 心

xinyuan 心猿

Xingxiang $z i$ 行香子

xiuxing jue 修行訣

Xiuxing wugeng song

修行五更頌

Xiuzhen shishu 修真十書

Xuanyao pian 玄要篇

Yang Mingzhen 楊明真

Yang zhenren 楊冝人

yi 意

Yifeng tang 藝風堂

yigeng chu一更初

yima意馬

Yin Zhiping 尹志平

Yu Ji 虞集

Yuqing jinsi qinghua biwen jinbao neilian danjue 玉清金筒青華秘文金寶 内鍊丹訣

(Yuzhi) Cipu (御製)詞譜

yunban 雲板

Yunji qiqian 雲笈七鐵

zaju 雜劇

Zhang Boduan 張伯端

Zhang Jixian 張繼先

Zhang Sanfeng xiansheng

quanji

張三丰先生全集

Zhang Yuchu 張宇初

zhenzhu珍珠

Zhuangzi jishi 莊子集釋

zi 子

zuowang mian 坐忘眠 


\title{
Vincent Goossaert
}

Sanshidai tianshi Xujing zhenjun yulu, Daozang 1249.

\section{Résumé}

Vincent GoossaERT : Poèmes taoïstes des cinq veilles

Les taoïstes ont toujours marqué leur méfiance vis-à-vis du sommeil, des rêves et du manque de contrôle sur soi qui s'ensuit. Dans l'ordre monastique Quanzhen, cette peur a donné naissance à une pratique ascétique de privation de sommeil et à une méditation nocturne collective. En liaison avec cette pratique, le Quanzhen a produit pendant les époques Jin et Yuan des « poèmes des cinq veilles » décrivant les étapes de la méditation pendant la nuit. Seize séries sont présentées, dont deux sont traduites en entier. L'auteur s' interroge sur le lien entre ce genre poétique bien précis, qui avait déjà été précédemment utilisé au sein du bouddhisme, et la pratique religieuse individuelle et communautaire des taoïstes Quanzhen. Il suggère que le récit d'une expérience spirituelle individuelle par ce moyen très normatif permet de l'insérer dans l'histoire de la communauté, mais aussi que les poèmes constituent en retour des guides pour l'adepte débutant.

\begin{abstract}
Vincent GoossaerT: Taoist Poems of the Five Night Watches

Taoists have always been suspicious of sleep, because dreams cause a loss of control over oneself. Within the Quanzhen monastic order, such a fear induced ascetical practices of sleep deprivation and motivated collective meditation at night. In this context appeared during the Jin and Yuan periods a number of « Poems of the Five Night Watches » describing night-time meditation. Sixteen sets of these poems are introduced, and two are translated entirely. The author questions the relationship between this given poetical genre (which had been used by Buddhists before) and the individual and collective practice of the Quanzhen Taoists. He suggests that narrating one's spiritual experience through this highly normative medium allows it to find its place within the religious community's history. The poems, in turn, may be used as guides for the beginner in meditation.
\end{abstract}

